\title{
Speciation and Bursts of Evolution
}

\author{
Chris Venditti • Mark Pagel
}

Published online: 5 June 2008

(C) Springer Science + Business Media, LLC 2008

\begin{abstract}
A longstanding debate in evolutionary biology concerns whether species diverge gradually through time or by rapid punctuational bursts at the time of speciation. The theory of punctuated equilibrium states that evolutionary change is characterised by short periods of rapid evolution followed by longer periods of stasis in which no change occurs. Despite years of work seeking evidence for punctuational change in the fossil record, the theory remains contentious. Further there is little consensus as to the size of the contribution of punctuational changes to overall evolutionary divergence. Here we review recent developments which show that punctuational evolution is common and widespread in gene sequence data.
\end{abstract}

Keywords Speciation · Evolution · Phylogeny ·

Punctuational evolution . Charles Darwin

"As natural selection acts solely by accumulating slight, successive, favorable variations, it can produce no great or sudden modifications; it can act only by short and slow steps" (Darwin 1859).

Understanding the range and diversity of organisms we see today is a fundamental challenge for biology. To meet this challenge, evolutionary biologists attempt to describe how and why evolutionary changes accumulate over time. Charles Darwin's 1859 theory of evolution by natural selection put forward the idea that evolutionary change or divergence accumulates steadily and slowly, in small steps, as one species succeeds the previous one in the struggle for existence. It was a revolutionary idea at the time, but now

C. Venditti $\cdot$ M. Pagel $(\bowtie)$

School of Biological Sciences, University of Reading,

Whiteknights,

Reading RG6 6AJ, UK

e-mail: m.pagel@reading.ac.uk
Darwin's gradualistic view of evolution has become widely accepted and deeply carved into biological thinking.

Over 110 years after Darwin introduced the idea of natural selection in his book The Origin of Species, two young paleontologists put forward a controversial new theory of the tempo and mode of evolutionary change. Niles Eldredge and Stephen Jay Gould's (Eldredge 1971; Eldredge and Gould 1972) theory of Punctuated Equilibria questioned Darwin's gradualistic account of evolution, asserting that the majority of evolutionary change occurs at or around the time of speciation. They further suggested that very little change occurred between speciation events - a phenomenon they referred to as evolutionary stasis.

Eldredge and Gould had arrived at their theory by studying patterns of evolution in the fossil record. It was well known even by Darwin that, when viewed in the fossil record, many species show rapid bursts of change that are often followed by longer periods in which little or no change occurs. However, detractors often argued that the appearance of punctuated change, and especially of stasis, was an artifact of the incompleteness or coarseness of the fossil record. The idea is that the vast geological time periods represented by fossil strata obscure many of the smaller changes that occur on a "real-time" basis. If so, the geological data will be of very low resolution, giving the appearance of stasis when in fact gradual changes have occurred (Hecht and Hoffman 1986; Hunt 2007). In reply, Eldredge and Gould maintained that patterns consistent with punctuational change were observed even when the fossil record was exceptionally good; this led them in 1977 to coin the phrase "stasis is data" (see Gould and Eldredge 1977), which has become something of a motto for their theory.

More than 30 years on and the theory of punctuated equilibria remains controversial, with many biologists still unconvinced as to the value of the theory as a description of 
how evolution proceeds. Much of the controversy surrounding it stems from the perception that Eldredge and Gould were in some way suggesting that new nonDarwinian evolutionary processes were required to explain punctuational evolution - that, somehow, the processes of change had become unhooked from the gradual tug of natural selection. These issues are discussed by Niles Eldredge (2008) in the article "The early 'evolution' of 'Punctuated Equilibria"' in a previous issue of this journal. In spite of these clarifications, and although attempts have been made to assess how common punctuated evolution is in the fossil record (see Erwin and Antsey 1995), there remains little consensus as to the generality of the theory.

Several years ago, we realized that there might be a way to investigate punctuational evolution without having to resort to observations taken from the fossil record. Our idea was that historical events of punctuational and gradual evolution should leave a distinctive signature on what biologists call phylogenetic trees. In the next two sections, we describe what a phylogenetic tree is and how biologists use them. We then go on to discuss how we employed them to search for evidence of punctuational bursts of evolution.

\section{Phylogenetic Trees}

In publishing his theory of evolution by natural selection, Charles Darwin showed that groups of species diverge over time from a common ancestral species by a process of what he called "descent with modification." Darwin was among the first to use the metaphor of a tree to illustrate this idea. In much the same way that we often draw family trees or genealogies to represent the lines of descent within a family, phylogenetic trees describe how, over evolutionary time, ancestral species give way to new descendant species. Darwin repeatedly used tree diagrams to convey his thoughts (Fig. 1), and as a measure of the importance he assigned them, the only figure in the Origin of Species depicts an idealized tree showing species, speciation and extinction events.

Today, biologists reconstruct evolutionary history to reveal how species are related to each other. Like Darwin, they represent this information in a tree-like form. The field of study for inferring phylogenies is called phylogenetics and the trees produced are called phylogenies or phylogenetic trees. The term phylogeny literally means the origin or genesis of groups. Up until recently, phylogenies were inferred from the analysis of the similarities and differences among species based on a suite of morphological characteristics. It is now relatively easy to obtain gene-sequence information, and genetic data have largely replaced morphological characters as the data of choice for inferring trees of contemporary species.

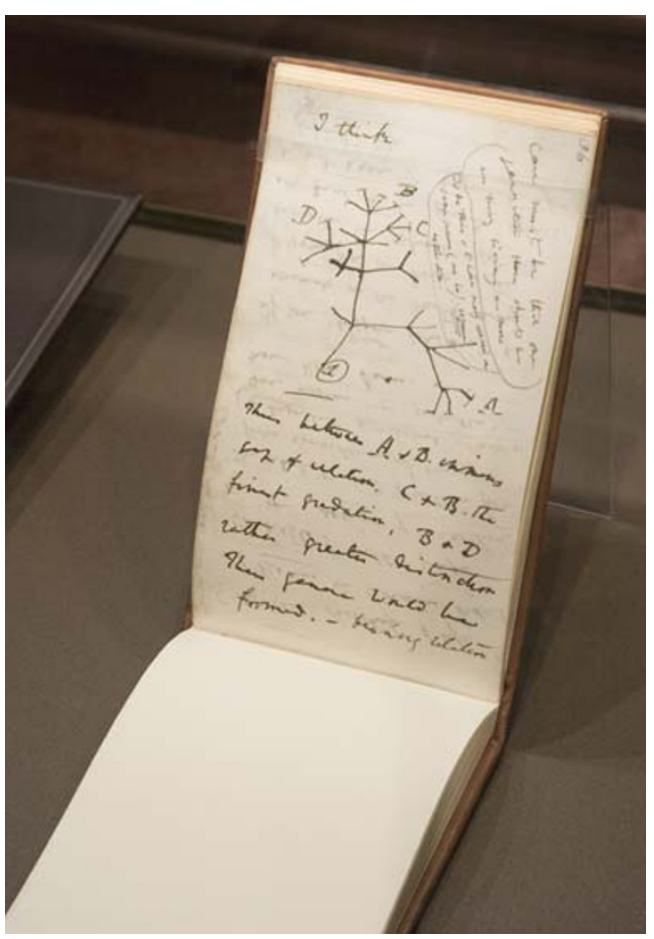

Fig. 1 Charles Darwin's first known sketch of a tree describing how organisms are related. The sketch appears in Darwin's first notebook on transmutation of species dating from 1837. (Image credit: (C) Auckland Museum.)

Phylogenetic trees can tell us more about the patterns and process of evolution than is at first obvious. Phylogenies, by tracing the evolutionary history of a group of organisms over a long period of time, can be used to infer the historical processes of evolution that gave rise to the diversity we see today. The nodes or points on the tree where a split occurs represent the historical event of an ancestral species giving rise to daughter or descendant species. The branches between these nodes represent periods of evolution between speciation events. It is natural to think of these branches as recording the amount of time between speciation events. However, if the phylogeny is constructed from gene-sequence data, the length of the branches records the amount of molecular or gene-sequence evolution that has occurred over the time period the branch represents (Fig. 2); that is, it records how different in an evolutionary genetic sense the daughter species is from its ancestor. In a previous issue of this journal, Gregory (2008) provides a more in-depth explanation of phylogenic trees and how to interpret them.

\section{Punctuated Molecular Evolution}

The possibility of a link between the rate (or amount) of evolution and speciation has interested biologists for over 50 years (Simpson 1944; Mayr 1954). In contrast to a 


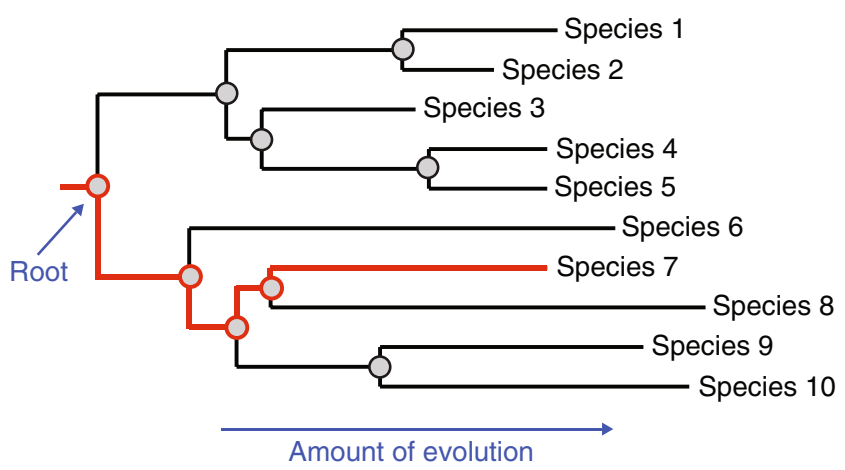

Fig. 2 A phylogenetic tree, the filled circles are called nodes and represent an event of speciation in which - moving from left to rightone species becomes two. The horizontal branches measure the amount of genetic evolution between speciation events. The vertical lines have no meaning and are just for spacing. What we refer to as a 'path' (see text) is highlighted in red through the tree. The amount of evolution along a path (sum of the horizontal branches) is called the path length. The red circles identify the speciation events along that path

gradualistic view of evolutionary change, the existence of a link might suggest some special role for speciation per se as a contributor to the modification part of Darwin's descent with modification. It would also form part of the expected signature of punctuational evolution.

A few years ago, we realized it would be possible to detect whether short or punctuational episodes of evolution had occurred in the genes used to infer phylogenetic trees (Webster et al. 2003). For each species represented in a phylogenetic tree, we can measure two values. The first is the total amount of evolution that has occurred between the base or root of the tree and the modern species alive today. The base of the tree represents the common ancestor of all the modern or extant species. We call the amount of evolution that has occurred along each of the paths leading to the modern species, the total path length, and we calculate it as the sum of all the individual branches along the lineage. Bearing in mind that the branches record the amount of genetic change between successive species, the total path length records the amount of genetic change that

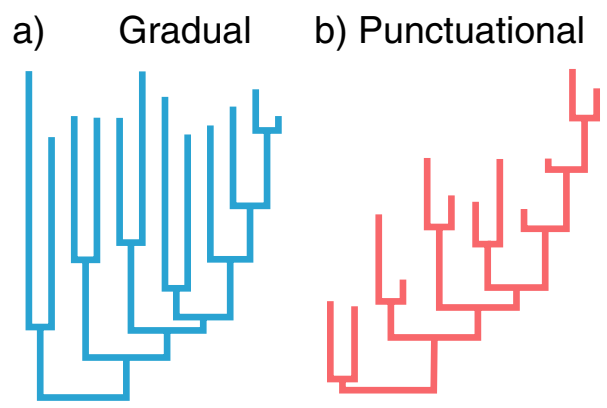

Fig. 3 Signatures of punctuational and gradual evolution on phylogenetic trees. a A phylogenetic tree which shows the signature of gradual evolution. That is, the total amount of evolutionary change, as measured by the path lengths, is not related to the number of speciation events that have occurred (see Fig. 2 caption for definition separates the common ancestral species from its descendants. The second value we record is the number of nodes or historical splitting events along each path through the tree. Some lineages, such as many birds or monkeys living today, have gone through many speciation events since their respective common ancestors. By comparison, it would be interesting if, for example, species we think of today as "living fossils" have had few speciation events since the time of their last ancestor millions of years ago.

Figure 2 shows how the two values that we measure can be obtained from a phylogenetic tree. If evolution proceeds gradually and independently of speciation events, there would be no association or correlation between the number of nodes (historical speciation events) and path length (see Fig. 3a,c). That is, if the process of speciation plays no role itself in causing change, then we do not expect that modern species at the end of paths through the tree with more speciation events will have diverged more from their common ancestor at the base of the tree. If, on the other hand, speciation events are associated with an increase in the rate of evolution, we expect paths with more nodes along them to be longer; where there has been more speciation, more total genetic evolution will have accumulated (see Fig. 3b,c).

Our research group has applied this methodology to a large number of phylogenetic trees to search for evidence of punctuational evolution (Webster et al. 2003; Pagel et al. 2006). In one study, we collected information on the amount of gene-sequence evolution from 122 different sets of species. From these datasets, we constructed phylogenetic trees and examined them for a correlation between the number of speciation events and the amount of molecular evolution. The trees included in our study describe the evolution of organisms as diverse as beetles, mushrooms, rodents and roses. We found that, in $35 \%$ of trees we studied, there was evidence for bursts of evolution in genes associated with events of speciation - the signature of punctuational evolution. Figure 4 shows a real phylogeny

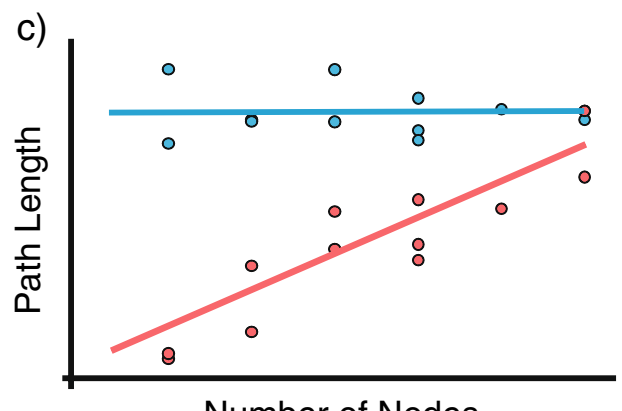

Number of Nodes

of terms, see blue line and points in c). b A phylogenetic tree which shows the signature of punctuational evolution. Evolutionary change accumulates at speciation events. Path lengths correlate with number of nodes, see red line and points in $\mathbf{c}$ 


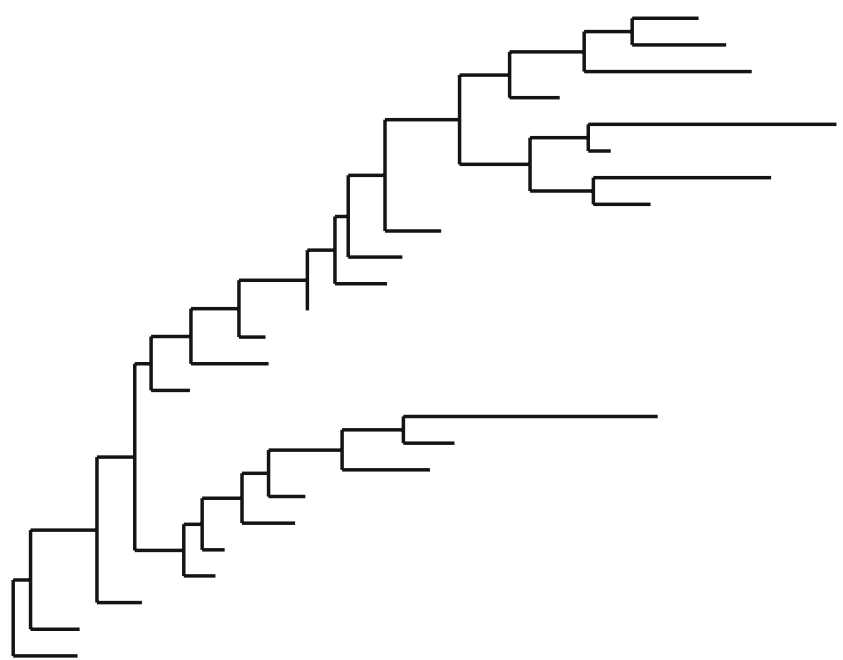

Fig. 4 A real phylogenetic tree of a group of endemic Hawaiian plants (that is, these plants are only found in Hawaii) which shows the distinctive pattern of punctuational molecular evolution. It is clear that in lineages with more nodes along them there is more evolution. Figure adapted from Webster et al. (2003)

of a group of endemic Hawaiian plants that displays the distinctive pattern of punctuational evolution. We were also able to examine whether punctuational effects were more common in some kinds of organisms than others. We found that punctuational changes were more common in plants $(57 \%)$ and fungi (71\%) than in animals (21\%).

An objection that might be raised about our findings is that rather than indicating an effect of speciation on rates of evolution, the reverse could be true. That is, maybe it is the case that lineages within our phylogenies that have, for whatever set of reasons, higher rates of evolution speciate more often. This would give the pattern of more speciation being associated with more evolution but would say nothing about punctuational change. We cannot strictly rule out this objection but, as we have discussed elsewhere (Pagel et al. 2006), we think it unlikely. Evolutionary biologists have, for decades, sought evidence for traits associated with increased rates of evolution. For example, we might suspect that animals with shorter generation times will evolve more quickly because they reproduce many times for every time that an animal with a longer lifespan does. Very few examples of such traits have been discovered, and in our case we would not expect such traits to differ among the species within a given phylogenetic tree. This is because our trees describe groups of closely related species that tend to be very similar in such background characteristics as generation time or body size and measures of metabolism. For these reasons, we think our analyses point to speciation being the cause of the changes we measure.

Using our phylogenetic method for studying punctuated evolution, it is also possible to calculate how much of the total amount of evolution can be attributed to the bursts associated with speciation events. Each branch of a phylogeny is the product of two processes; some proportion of the evolution is punctuational and some is gradual. We were able to show that on average $22 \%$ of the evolution is attributable to the punctuational burst, with the remainder accumulated gradually. Despite there being significant differences in the likelihood of punctuational evolution occurring among plants, fungi and animals, we found no difference in the size of the effect.

Our studies, therefore, suggest that rapid bursts of evolution associated with speciation events represent an important, previously underappreciated contributor to evolutionary divergence. Interestingly, we find no genetic counterpart to the phenomenon of evolutionary stasis, the other part of Eldredge and Gould's punctuated equilibrium theory. That is, if around $22 \%$ of the total evolution arises in bursts, somewhere around $78 \%$ is accumulating by gradual means. This may not be surprising because we have studied genes, whereas Gould and Eldredge studied morphology. It is well known that some kinds of genetic evolution can occur without causing a change to the species' morphology. Evolutionary biologists call these changes neutral to signify that they do not produce measurable changes to the organism. By comparison, nonneutral or what are sometimes called coding changes are changes to genes that lead to measurable changes to organisms.

Our measures of genetic evolution include both of these kinds of change. There is good reason to expect that there will be gradual changes to the coding parts of genes during the long periods of time between speciation events. The coding parts of genes often evolve slowly, so we might expect these changes to be relatively small in number and consequently so might be their influence on the outward form of the organism. By comparison, neutral changes occur in the background all of the time, at a higher rate and independently of whether a species is changing its outward appearance or phenotype. There is reason to expect that these changes will be greater in number than the coding changes. Therefore, the periods of stasis in morphological traits that Eldredge and Gould and others have observed may correspond to periods of predominantly neutral changes in the genes we have studied.

In this sense, the $22 \%$ figure associated with speciation is, we believe, large. This is because we think the punctuational episodes are relatively short periods in the lifetime of a species and yet as much as $22 \%$ of the total genetic change is occurring in them. It may be possible, in the future, to untangle the evolutionary genetic changes that cause a change to the phenotype and those that do not and study these individually for their contribution to punctuational evolution. At the same time, as it stands, it is important to be clear that our results do not provide either evidence for or against stasis at the morphological level. 


\section{Mechanisms for Bursts of Evolution}

How are we to reconcile these punctuational bursts with conventional Darwinian accounts of evolution? Two wellestablished mechanisms for speciation may provide answers. One makes use of what biologists call founder effects. The other invokes rapid evolutionary change as species enter new niches. When a species acquires a trait that allows it to exploit some new feature of the environment, it can be said that it occupies a new environmental niche. The species may then show rapid changes in other traits as it adapts to this new niche.

Small populations sometimes break from a large ancestral one and form a new population. This might happen if some of the individuals in a group move to a new area or if some geological event occurs to separate them. If the separation between the two is maintained, in time this new population may form a new species. In this situation, chance can play an important role in which alleles or variant forms of a gene are represented in this new population. In most populations there will be several different forms or alleles of the same gene found among its members. If a new population is formed from a small sample the proportion of alleles present can be different from the ancestral group. Think of a group of individuals wearing red or white hats where the colour of a hat signifies the presence of a particular variant of a gene. If we randomly sampled a small number of these individuals, all might have the same hat color. But if we sampled a large number of them, we would almost certainly include people with hats of both colors.

In small populations, an allele is more likely simply as a result of chance to go to fixation-all members of the population will come to have it - than the same allele in a large population. This process of the random fixation of alleles is known as genetic drift and it is one of the ways that isolated groups of organisms acquire genetic differences. In 1954, the well-known evolutionary biologist Ernst Mayr proposed that speciation often occurs following the formation of small isolated populations. In a recent edition of this journal (January 2008), Anastasia Thanukos reviews and explains Mayr's ideas on speciation.

For our purposes, it is intriguing that the effect of drift is greatest in small groups and rapidly declines as population size increases. If, when new species form, they often begin as a small and isolated population, this gives us the ingredients for a punctuational pattern of evolution. That is, we expect that for a short period of time the rate of evolution will be accelerated owing to the effects of chance and genetic drift. This will be followed by a return to the "normal" or background rate of evolutionary change as the new species grows in numbers. This mechanism can only work if migration and interbreeding between the ancestral population and the new founder population is extremely low or even nonexistent: some population geneticists think even "extremely low" migration and interbreeding are enough to prohibit speciation by founder effects.

The second general mechanism is adaptive evolution as species invade a new ecological niche. Consider, for example, that a population of a moth species finds itself living among flowers or plants of a different color to those normally encountered by the original or ancestral moth population. We might expect natural selection to favor individual moths in this new population whose appearance better matches the background, as they will avoid predation. Over time, this process may cause the population to become a new, and differently camouflaged, species. If occupying a new or different niche commonly attends speciation, then we might expect to see a transient increase in rate of evolutionary change as this adaptive process occurs. This increase is transient because over the longer term, as the new species becomes better adapted to its new surroundings, the rate will slow.

Both these mechanisms rely on fast and continued reproductive isolation. This means the two populations stop interbreeding (migration between them is very low). Recently, evolutionary biologists have found that rapid reproductive isolation is more common than previously thought (Culotta and Pennisi 2005) and it is often associated with what is known as sympatric speciation or speciation between populations which share the same geographical range. Many of the mechanisms which can cause this rapid reproductive isolation are more common in plants and perhaps fungi than animals and therefore may go some way in explaining the differences we have observed in the frequency of punctuational evolution among these groups.

Looking around us, it may be that punctuational bursts of evolution are responsible for the surprising morphological diversity between some very closely related groups. Among the 80 or so species of the Andean genus Lupinus (Hughes and Eastwood 2006) - a flowering plant directly related to the common garden Lupine- some are over $12 \mathrm{ft}$ $(3.7 \mathrm{~m})$ tall while others are barely bigger than 2 in. $(5 \mathrm{~cm})$. Some are tree like; others are herbs and others yet take the form of small bushes. Similarly, Lake Tanganyika in central Africa supports a diverse radiation of the cichlid fish of the genus Tropheus (Egger et al. 2007). This radiation, beginning just one million years ago, is thought to have produced the numerous lineages, with diverse morphology, we see today.

Detecting punctuational evolution at the molecular level, then, does not mean that Darwin got it wrong; likewise, it does not require a reevaluation of modern evolutionary theory. Conventional neo-Darwinian ideas such as natural selection and random drift can explain these bursts of 
evolution. What is interesting about punctuational effects is that speciation itself may be an important factor in increasing the rate of evolutionary change. The evolutionary changes could still accumulate in small steps, but these steps are taken more quickly at the time of speciation.

\section{Punctuated Language Evolution}

"The formation of different languages and of distinct species, and the proofs that both have been developed through a gradual process, are curiously parallel" (Darwin 1871)

As is often the case with matters of evolution, Charles Darwin was among the first to realize that language and species might evolve in a similar way. Researchers today generally agree that many of the principal characteristics of linguistic evolution are analogous to those of species or biological evolution. In a similar way to morphological characters and gene sequences of species, languages have heritable units that can be passed to subsequent generations and may be subject to forces similar to natural selection, mutation and genetic drift (Atkinson et al. 2008; Croft 2000).

It is possible to derive a phylogenetic tree of languages based on the similarities and differences in vocabulary. Such trees can inform us about the evolutionary history of a particular language family. In these trees, the branches that separate the nodes are measured in units of lexical replacement (acquisition of novel words); the nodes themselves represent language-splitting events, the linguistic equivalent to a speciation event.

We recently (Atkinson et al. 2008) sought evidence for punctuational bursts of linguistic evolution in phylogenetic trees inferred from vocabulary data using three major language families - Bantu (a large category of African languages), Indo-European and Austronesian. Together, these account for more than one third of the world's languages. We found evidence in all cases for punctuational bursts of language evolution associated with the formation of new languages. Further, a surprising amount of the overall lexical divergence among languages was attributable to punctuational effects: $31 \%$ of the vocabulary differences among Bantu languages occurred at splitting events, $21 \%$ in Indo-European languages and $10 \%$ in Austronesian.

The rate of language evolution and factors affecting it has been discussed by linguists for many years. Kirch and Green (1987) suggested that the movement of Polynesians inhabiting Pacific islands might have caused an increased rate of language evolution owing to the many and successive founder events to which such island hopping would have led. These founder events are the linguistic equivalent of genetic founder events. The Polynesian languages are a subset of the Austronesian tree. When the punctuational test was carried out on the Polynesian subtree, the contribution of punctuational effects increased to $33 \%$, over three times that of the Austronesian tree taken as a whole!

It seems, then, that at least some punctuational bursts of language evolution may arise from founder effects. However, language may be useful for things other than mere communication. Linguists have long viewed language as a facilitator for group cohesion and identity. This being the case, the punctuational language change observed may reflect humans' capacity to adjust languages at critical times in our cultural evolutionary history; maintaining cohesion in the light of the emergence of new and/or rival groups. We note that Noah Webster, who created the first dictionary of American English, deliberately introduced spelling differences between American and British English, asserting that "[A]s an independent nation, our honor requires us to have a system of our own, in language as well as government" (Webster 1789). These new spellings appeared almost instantaneously and have persisted to this day.

\section{New Directions}

Speciation frequently seems to act as a driving force for molecular evolution, a phenomenon that has often been overlooked by biologists. Further, the analysis of language data shows that the search for punctuational effects can also move outside biology. What are traditionally biological techniques, including phylogenetics, are gaining momentum in other disciplines, such as anthropology and archaeology. Ford (1962) proposed that cultural change is often reflected in cultural artifacts, and he illustrated the point by noting changes in ceramic vessels over time. Phylogenetic trees have now been constructed for many material cultural artifacts including spear heads (O'Brien et al. 2001), textiles (Tehrani and Collard 2001) and musical instruments (Temkin and Eldredge 2007). It has even been suggested that phylogenetic trees can be derived from songs and/or music (Fitch 2005; Fitch 2006).

In principal, cultural phylogenetic trees could be studied for punctuational evolution. If the evolution of the cultural artifacts is characterised by descent with modification, such analyses would be justified. In fact, if a meaningful phylogeny can be inferred from data derived from them, it goes some way in showing that cultural evolution often proceeds in a manner analogous to biological evolution.

The theory of punctuated equilibrium was originally proposed to explain morphological evolution observed in the fossil record. To our knowledge, there is no phyloge- 
netic research that has demonstrated bursts of morphological evolution, but the methodology we have used is new and we look forward to investigators applying it to morphological characters in the future. The study of punctuational evolution represents an area of great potential research which might connect disparate branches of evolutionary biology and perhaps disciplines beyond.

Acknowledgements We thank Niles Eldredge for inviting us to submit this article. Our research is supported by grants to MP from the Natural Environment Research Council of the United Kingdom and the Leverhulme Trust.

\section{References}

Atkinson QD, Meade A, Venditti C, Greenhill SJ, Pagel M. Languages evolve in punctuational bursts. Science 2008;319:588.

Culotta E, Pennisi E. Breakthrough of the year: evolution in action. Science 2005;310:1878-9.

Croft W. Explaining language change: an evolutionary approach. Harlow: Pearson Education; 2000.

Darwin C. On the origin of species by means of natural selection, or the preservation of favoured races in the struggle for life. London: John Murray; 1859.

Darwin C. The decent of man. London: Murray; 1871.

Egger B, Koblmüller S, Sturmbauer C, Sefc KM. Nuclear and mitochondrial data reveal different evolutionary processes in the Lake Tanganyika cichlid genus Tropheus. BMC Evol Biol 2007;7:137-51.

Eldredge N. The allopatric model and phylogeny of Paleozoic invertebrates. Evolution 1971;25:156-67.

Eldredge N, Gould SJ. Punctuated equilibria: an alternative to phyletic gradualism. In: Schopf TM, editor. Models in palaeobiology. San Francisco: Freeman Cooper; 1972. p. 82-115.

Eldredge N. The early "evolution" of "punctuated equilibria". Evo Edu Outreach 2008;1:107-13.

Erwin DH, Antsey RL. Speciation in the fossil record. In: Erwin DH, Antsey RL, editors. New approaches to speciation in the fossil record. New York: Columbia University Press; 1995. p. 11-38.
Fitch WT. The evolution of music in comparative perspective. Ann N Y Acad Sci 2005;1060:1-21.

Fitch WT. The biology and evolution of music: a comparative perspective. Cognition 2006;100:173-215.

Ford JA. A quantitative method for deriving cultural chronology. Pan American Union Technical Bulletin No. 1. Washington, DC: Pan American Union; 1962.

Gould SJ, Eldredge N. Punctuated equilibria: the tempo and mode of evolution reconsidered. Paleobiology 1977;3:115-51.

Gregory TR. Understanding evolutionary trees. Evo Edu Outreach 2008; 1:121-37.

Hecht MK, Hoffman A. Why not neo-Darwinism? A critique of paleobiological challenges. In: Dawkins R, Ridley M, editors. Oxford surveys in evolutionary biology. Oxford: Oxford University Press; 1986. p. 1-48.

Hughes CE, Eastwood RJ. Island radiation on a continental scale: exceptional rates of plant diversification after uplift of the Andes. Proc Natl Acad Sci 2006;103:10334-9.

Hunt G. The relative importance of directional change, random walks, and stasis in the evolution of fossil lineages. Proc Natl Acad Sci 2007; 104:18404-8.

Kirch PV, Green RC. History, phylogeny, and evolution in Polynesia. Curr Anthropol 1987;33:161-86.

Mayr E. Change of genetic environment and evolution. In: Huxley J, Hardey AC, Ford EB, editors. Evolution as a process. London: Allen and Unwin; 1954. p. 157-80.

O'Brien MJ, Darwent J, Lyman RL. Cladistics is useful for reconstructing archaeological phylogenies: Palaeo-Indian points from the southeastern United States. J Archaeol Sci 2001;28:1115-36.

Pagel M, Venditti C, Meade A. Large punctuational contribution of speciation to evolutionary divergence at the molecular level. Science 2006;314:119-21.

Simpson GG. Tempo and mode in evolution. New York: Columbia University Press; 1944.

Tehrani J, Collard M. Investigating cultural evolution through biological phylogenetic analyses of Turkmen textiles. J Anthropol Archaeol 2001;21:443-63.

Temkin I, Eldredge N. Phylogenetics and material cultural evolution. Curr Anthropol 2007;48:146-53.

Webster AJ, Payne RJ, Pagel M. Molecular phylogenies link rates of evolution and speciation. Science 2003;301:478.

Webster N. Dissertations on the English language. Boston: Isaiah Thomas; 1789. 